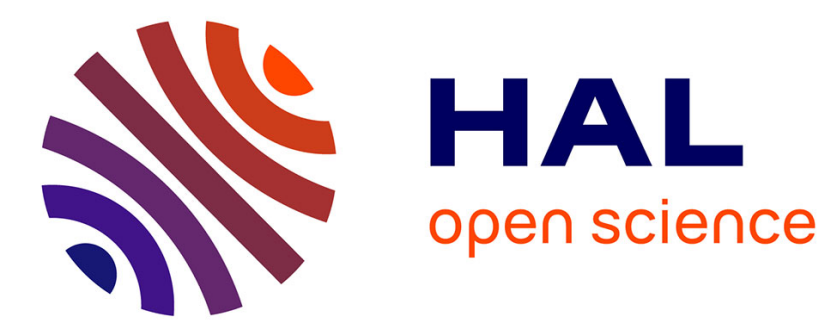

\title{
Microtubule-induced nuclear envelope fluctuations control chromatin dynamics in Drosophila embryos
}

Bernhard Hampoelz, Yannick Azou-Gros, Roxane Fabre, Olga Markova, Pierre-Henri Puech, Thomas Lecuit

\section{To cite this version:}

Bernhard Hampoelz, Yannick Azou-Gros, Roxane Fabre, Olga Markova, Pierre-Henri Puech, et al.. Microtubule-induced nuclear envelope fluctuations control chromatin dynamics in Drosophila embryos. Development (Cambridge, England), 2017, 144 (4), pp.732. 10.1242/dev.149575 . hal-01765088

\section{HAL Id: hal-01765088 \\ https://hal-amu.archives-ouvertes.fr/hal-01765088}

Submitted on 26 Oct 2018

HAL is a multi-disciplinary open access archive for the deposit and dissemination of scientific research documents, whether they are published or not. The documents may come from teaching and research institutions in France or abroad, or from public or private research centers.
L'archive ouverte pluridisciplinaire HAL, est destinée au dépôt et à la diffusion de documents scientifiques de niveau recherche, publiés ou non, émanant des établissements d'enseignement et de recherche français ou étrangers, des laboratoires publics ou privés.

\section{(c)(1)}

Distributed under a Creative Commons Attribution| 4.0 International License 


\section{Correction: Microtubule-induced nuclear envelope fluctuations control chromatin dynamics in Drosophila embryos}

Bernhard Hampoelz, Yannick Azou-Gros, Roxane Fabre, Olga Markova, Pierre-Henri Puech and Thomas Lecuit

The incorrect affiliation was listed for Pierre-Henri Puech. The correct affiliation is INSERM UMR 600/CNRS UMR 6212, Case 937-163, Avenue de Luminy, 13288 Marseille Cedex 09, France.

The authors apologise to readers for this mistake. 


\title{
Microtubule-induced nuclear envelope fluctuations control chromatin dynamics in Drosophila embryos
}

\author{
Bernhard Hampoelz ${ }^{1}$, Yannick Azou-Gros ${ }^{1}$, Roxane Fabre ${ }^{2}$, Olga Markova ${ }^{1}$, Pierre-Henri Puech ${ }^{1}$ and \\ Thomas Lecuit ${ }^{1, *}$
}

\begin{abstract}
SUMMARY
Nuclear shape is different in stem cells and differentiated cells and reflects important changes in the mechanics of the nuclear envelope (NE). The current framework emphasizes the key role of the nuclear lamina in nuclear mechanics and its alterations in disease. Whether active stress controls nuclear deformations and how this stress interplays with properties of the NE to control NE dynamics is unclear. We address this in the early Drosophila embryo, in which profound changes in NE shape parallel the transcriptional activation of the zygotic genome. We show that microtubule (MT) polymerization events produce the elementary forces necessary for NE dynamics. Moreover, large-scale NE deformations associated with groove formation require concentration of MT polymerization in bundles organized by Dynein. However, MT bundles cannot produce grooves when the farnesylated inner nuclear membrane protein Kugelkern (Kuk) is absent. Although it increases stiffness of the NE, Kuk also stabilizes NE deformations emerging from the collective effect of MT polymerization forces concentrated in bundles. Finally, we report that MT-induced NE deformations control the dynamics of chromatin and its organization at steady state. Thus, the $\mathrm{NE}$ is a dynamic organelle, fluctuations of which increase chromatin dynamics. We propose that such mechanical regulation of chromatin dynamics by MTs might be important for gene regulation.
\end{abstract}

KEY WORDS: Chromatin, Drosophila, Microtubules, Nuclear envelope

\section{INTRODUCTION}

Although nuclei are often spherical, nuclear shape can change significantly between cell types and during cell differentiation or aging (Dauer and Worman, 2009). The nuclear envelope (NE) is a key determinant of nuclear morphology. The nuclear lamina, a meshwork underlying the inner nuclear membrane in metazoa, is crucial for the structural integrity of nuclei (Stuurman et al., 1998). Lamins, the major constituents of the lamina, not only support the structure of nuclei but also play important roles in transcription, replication or chromatin organization (Dechat et al., 2008). In most species there are two different types of lamins: a ubiquitously expressed B-type lamin, and one or more A-type lamins, which become only expressed when cells start to differentiate. A-type lamins control the mechanics of the NE and are responsible for the increased stiffness of nuclei in differentiated cells compared with stem cells (Pajerowski et al., 2007). Thus, nuclear mechanics must be tightly regulated. Changes in nuclear mechanics are accompanied by defects in gene expression and a distorted nuclear morphology (Dahl et al., 2008; Dauer and Worman, 2009; Lammerding et al., 2004). It is thus essential to decipher the mechanisms regulating nuclear mechanics and morphogenesis. By analogy to cell shape changes controlled by generators of forces and the mechanical response of the cortex, we sought to investigate the origins of nuclear deformations and to delineate active forces that shape nuclei and the mechanical properties of the NE.

${ }^{1}$ IBDML, UMR6216 CNRS-Université de la Méditerranée, Campus de Luminy, Case 907, 13288 Marseille Cedex 09, France. ${ }^{2}$ INSERM UMR 600/CNRS UMR 6212, Case 937-163, avenue de Luminy, 13288 Marseille Cedex 09, France.

*Author for correspondence (thomas.lecuit@ibdml.univmed.fr)
We address this in the physiological context of the early Drosophila embryo, in which profound changes in nuclear shape parallel transcriptional activation of the zygote. After 13 rounds of mitosis without cytokinesis, somatic nuclei at the embryo periphery are packaged by the invaginating plasma membrane (PM) during interphase 14, a process called cellularization (Schejter and Wieschaus, 1993). Nuclei are regular and spherical when cellularization starts, but subsequently elongate into ellipsoids and acquire an irregular, lobulated morphology (Fig. 1A,B) (Brandt et al., 2006; Pilot et al., 2006). While nuclear elongation depends on microtubules (MTs) (Edgar et al., 1987), it is largely unclear how the NE becomes lobulated. Yet it does not involve A-type lamin, which is expressed only later in development (Riemer et al., 1995). Instead, the farnesylated inner nuclear membrane (INM) protein Kugelkern [Kuk; also known as Charleston (Char)], which is strongly upregulated at the onset of cellularization, is required for NE morphogenesis (Brandt et al., 2006; Pilot et al., 2006). Similar to membrane anchored lamins, ectopic expression of Kuk exacerbates NE lobulation in flies (Brandt et al., 2008) and also in other systems (Polychronidou et al., 2010). How Kuk regulates NE morphogenesis is not understood.

Nuclear morphogenesis occurs concomitantly with the upregulation of the zygotic genome. In animals, this key developmental switch involves a massive upregulation of transcription as well as major rearrangements in chromatin structure and marks the onset of differentiation (Rudolph et al., 2007). In kuk mutants, transcription of certain zygotic genes is changed (Brandt et al., 2006). This suggests that nuclear morphology could contribute to gene expression.

Here, we investigate the mechanisms of NE morphogenesis by focusing on the dynamics of the NE, which is expected to reflect the presence of force generators. We demonstrate that nuclear 
deformation during Drosophila cellularization results from a combination of active cytoplasmic stresses and developmentally controlled properties of the NE. Finally, we show that the dynamics of the NE during cellularization promotes chromatin mobility and steady-state organization during zygotic induction.

\section{MATERIALS AND METHODS}

\section{Fly strains and antibodies}

The following fly lines were used: yw; UAS-EB1::GFP, mattubGAL4VP16/CyO (Jankovics and Brunner, 2006), yw sqh[AX3];sqhSqh::GFP (gift of R. Karess, Institut Jacques Monod, Université Paris Diderot, Paris, France), H2Av::GFP (gift of G. Cavalli, Institut de Génétique Humaine, CNRS, Montpellier, France), tub::GFP (full genotype: $w^{1118}$; P\{GAL4::VP16-nos, UTR $\} C G 6325^{M V D 1}, P\{U A S p$ GFPS65C-aTub84B\}3) (BL-7553), UAS::Dm0 (gift from C. Maurange, IBDML, Marseille, France), kuk (Brandt et al., 2006), 6xkuk (Brandt et al., 2006), $\mathrm{y}^{1} \mathrm{w}^{67 \mathrm{c} 23} ; P\{E Y 2 g\} C G 3287^{E Y 03560}$ (BL-20000). $\mathrm{w}^{1118}$ $\mathrm{Df}(2 \mathrm{R})$ CG3287-klaroid/CyO (Technau and Roth, 2008) yw flies were used as wild-type controls.

The following antibodies were used: mouse anti- $\alpha$ Tubulin (1:500, Sigma), mouse anti-acetylated Tubulin (1:500, Abcam), rabbit antiH3K4diMe (1:500, Upstate), mouse anti-HP1 [1:50, Developmental Studies Hybridoma Bank (DSHB)], rabbit anti-scribble (1:2500). For immunofluorescence experiments, embryos were dechorionated, washed and fixed in 4\% paraformaldehyde (PFA) and devitellinized by methanol popping. For anti- $\alpha$ Tubulin stainings, embryos were fixed for 1 minute in $37 \%$ PFA.

Injections were performed with Colcemid (1.25 mM, Sigma), Taxol (Paclitaxel, $20 \mathrm{mg} / \mathrm{ml}$, Sigma), WGA-Alexa488 or WGA-Alexa555 (100 $\mu \mathrm{g} / \mathrm{ml}$, Molecular Probes) and the following antibodies: mouse anti-Dhc [monoclonal antibody against the HUV fragment from Dhc (Sharp et al., 2000) $(6 \mathrm{mg} / \mathrm{ml})]$, mouse anti-Dic $(300 \mu \mathrm{g} / \mathrm{ml}$, Millipore), mouse anti-GST $(300 \mu \mathrm{g} / \mathrm{ml}$, Eurogentec). dsRNAi probes for $k u k$ and slam were injected at $5 \mu \mathrm{M}$.

\section{Embryo injections and live imaging}

Staged blastoderm embryos were treated as described (Cavey and Lecuit, 2008) and injected with fluorescently labeled WGA, drugs or neutralizing antibodies. Subsequently, embryos were filmed during interphase 14 using a $100 \times / 1.3$ oil objective on a Zeiss LSM 510 confocal microscope. 3D reconstruction was carried out using $0.5 \mu \mathrm{m}$ distant $z$-sections using the 3D segmentation editor of Amira. Nuclear surfaces were calculated with the 3D Virtual Embryo software (Tassy et al., 2006).

\section{Quantifications and statistics}

For quantification of NE dynamics, staged embryos injected with fluorescently labeled WGA were imaged at $50 \%$ nuclear height every 5 seconds for 6 minutes. Correction for drift was carried out by stack-cross correlation in EMBL ImageJ. Large-scale NE deformations were measured as the distance between the most lateral positions of the signal on a $2.5 \mu \mathrm{m}$ wide kymograph spanning the WGA signal. For high-frequency fluctuations, pixel positions of the WGA signal for each frame along linescans were determined in Matlab and standard deviations of the lateral position of the WGA maxima within 1-minute intervals were calculated.

Tub::GFP intensities were measured from confocal $z$-sections over whole nuclei at distinct time points during cellularization. Tub::GFP intensities at NE or cytoplasmic regions were determined from linescans recorded in MetaMorph at one $z$-plane at $50 \%$ nuclear height. Mean intensity values were normalized to the sum of NE and cytoplasmic tub::GFP intensity for each time point. To quantify tub::GFP intensities after antibody injections, ratios between NE-attached and cytoplasmic tub::GFP intensities were calculated inside or outside the WGA-labeled injection region, respectively.

To measure the lifetime of MT bundles, nuclei of tub::GFP-expressing embryos were imaged at one $z$-plane every 400 mseconds for 100 seconds. Kymographs along the NE were created in MetaMorph allowing measurement of bundle-persistence times.
To measure persistence times of H2Av::GFP spots, nuclei were imaged for 260 seconds with an acquisition every 2 seconds. Kymographs spanning entire nuclear diameters (see Fig. 6D) were recorded using MetaMorph and the length of H2Av::GFP tracks was measured in pixels.

All kymographs in the study represent fluorescence intensities of the respective signal where blue corresponds to low and red to high fluorescence (Fig. 1E).

To account for non-Gaussian distribution of datasets, (two-tailed) $P$ values were calculated by Mann-Whitney $U$-tests.

\section{Photobleaching experiments}

Photobleaching experiments were performed on a Zeiss 510 Meta confocal microscope with a $100 \times / 1.3$ oil objective. After ten pre-bleach images, twenty iterations at $100 \%$ transmission of a $488 \mathrm{~nm}$ Argon laser $(5.2 \mu \mathrm{W}$ photonic output power) were used to photobleach ROI between 1.6 to 6 $\mu \mathrm{m}^{2}$. Images were typically acquired at a pixel time of $6.4 \mu$ seconds and a scan frame of $128 \times 100$ pixels for 100 seconds. Fluorescence intensities were analyzed using MetaMorph. Raw fluorescence intensity measurements were background subtracted and normalized to arbitrary fluorescence units (pre-bleach time points normalized to one). Fluorescence recovery was calculated as the percentage of the bleached fluorescence obtained at a plateau.

\section{Isolation of nuclei for AFM}

Staged dechorionated embryos were lysed in lysis buffer $[25 \mathrm{mM}$ Tris $\mathrm{pH}$ 8.0, $27.5 \mathrm{mM} \mathrm{NaCl}, 20 \mathrm{mM} \mathrm{KCl}, 25 \mathrm{mM}$ sucrose, $10 \mathrm{mM}$ EDTA, $10 \mathrm{mM}$ EGTA, 10\% (v/v) glycerol, $0.5 \%$ Nonidet P40] and nuclei were isolated by centrifugation at $7000 \mathrm{rpm}(4500 \mathrm{~g})$ for 15 minutes. After resuspension in $20 \mu \mathrm{l}$ lysis buffer, nuclei were seeded onto a chamber delimitated by a plastic ring on a Hellmanex and plasma activated microscope slide. Before seeding, chambers were pre-incubated with WGA-Alexa488 $(20 \mathrm{mg} / \mathrm{ml})$ for up to 2 hours. Subsequently, nuclei could immobilize for $30-45$ minutes.

\section{Atomic force microscopy}

Experiments were conducted with an atomic force microscope (AFM; Nanowizard I, JPK Instruments, Berlin, Germany) mounted on an inverted fluorescence microscope (Zeiss Axiovert 200 equipped with $10 \times$ and $20 \times$ lens) (Puech et al., 2005). The AFM head was equipped with a $15 \mu \mathrm{m} z$ range linearized piezoelectric ceramic scanner and an infrared laser. The setup was used in closed height feedback mode (Puech et al., 2006). Before each experiment the optical lever system was calibrated on glass substrates and the cantilever spring constant was determined in situ using built-in routines of the JPK software by using the thermal noise method (Franz et al., 2007). Spring constants were found to be consistent with the manufacturer's nominal value (MSCT/MLCT, Veeco Instruments, nominal constant $10 \mathrm{pN} / \mathrm{nm}$ ). Experiments were run at $25^{\circ} \mathrm{C}$ in lysis buffer for a maximum of 1 hour.

The speed for placing or removing the tip at nuclei was set to 1 $\mu \mathrm{m} / \mathrm{second}$ and the desired contact force to $500 \mathrm{pN}$. Over a travel distance of $4 \mu \mathrm{m}, 1024$ points per second were measured. In order to gain the E modulus, the apparent contact time was set to zero to minimize potential dissipation contributions. Each of at least ten successive force curves per nucleus was examined by eye and processing was performed using the JPK-IP batch processing procedures: correcting for baseline shift and/or tilt for the pushing part of the force curve, finding the contact point between the tip and the nucleus, calculating the tip sample separation distance and fitting the pushing part of the force curve with the Hertz model (Schape et al., 2009) for a square-based pyramid with an average half angle of $21^{\circ}$ (calculated from the data of the provider). This last step allows the Young's modulus E to be gained (for a $1 \mu \mathrm{m} /$ second speed and $500 \mathrm{pN}$ indenting force). E values for each nucleus were pooled to calculate median values and quartiles. No correlation was observed for successive force curves on one nucleus or for successive nuclei.

For relaxation experiments, the contact time was set to 10 seconds and force was recorded versus time while the piezo position was kept constant. After reaching the preset contact force, nuclei relaxed the force in a pseudo-exponential way. One relaxation pattern was acquired per nucleus 
and the respective average relaxation was calculated for each condition. Standard deviations at each time point were similar for all three tested conditions and were larger than the spreading of the average curves.

\section{Particle image velocimetry}

H2Av::GFP-expressing embryos were imaged every 2 seconds in one medial $z$-plane for 260 seconds. Images were analyzed using the Particle Image Velocimetry free software program (MatPIV) and MatLab. MatPIV analysis is based on cross-correlation of intensity of small bins between subsequent time frames. Correction of horizontal displacement was carried out by subtraction of the average velocity. Spatial masks were applied to filter out vectors outside nuclei or internal regions by blurring by a Gaussian filter and the intensity threshold was applied for each image in the movie. For different genotypes or conditions, mean velocities were calculated for each frame and subsequently averaged.

\section{RESULTS}

\section{Microtubules control NE morphogenesis during} Drosophila cellularization

We focused our analysis on the dynamics of NE morphogenesis during early Drosophila development. For live analysis, the NE was labeled with fluorescent wheat germ agglutinin (WGA), a lectin that binds to a component of the nuclear pore complex (NPC) within the NE (Onischenko et al., 2004). As the NPC disassembles during mitosis, entry into interphase could be precisely timed by the reappearance of WGA at the nuclear rim. Our time-lapse analysis revealed that, starting at interphase 14, the NE remodels in a highly dynamic fashion (see Movie 1 in the supplementary material). NE dynamics was not restricted to cellularization but was also present in nuclei from gastrulating embryos or from third instar larvae wing discs (data not shown). We deciphered three phases in NE morphogenesis during cellularization: 12-15 minutes after mitosis, the NE lost its round shape and formed polygons, which later progressively acquired grooves during lobulation (see Movie 1 in the supplementary material; Fig. 1A,B-B"). The dynamics of groove formation was apparent in kymographs as NE lateral deformations (Fig. 1D,D'), which increased in amplitude as grooves formed $\left(P=7.23 \times 10^{-5}\right)$ (Fig. 1F). The formation of grooves could be a passive consequence of persistent NE growth during cellularization. However, measurements of the NE surface increase by 3D reconstruction from serial $z$-sections showed that $84 \%$ of surface increase preceded groove formation (see Fig. S1A in the
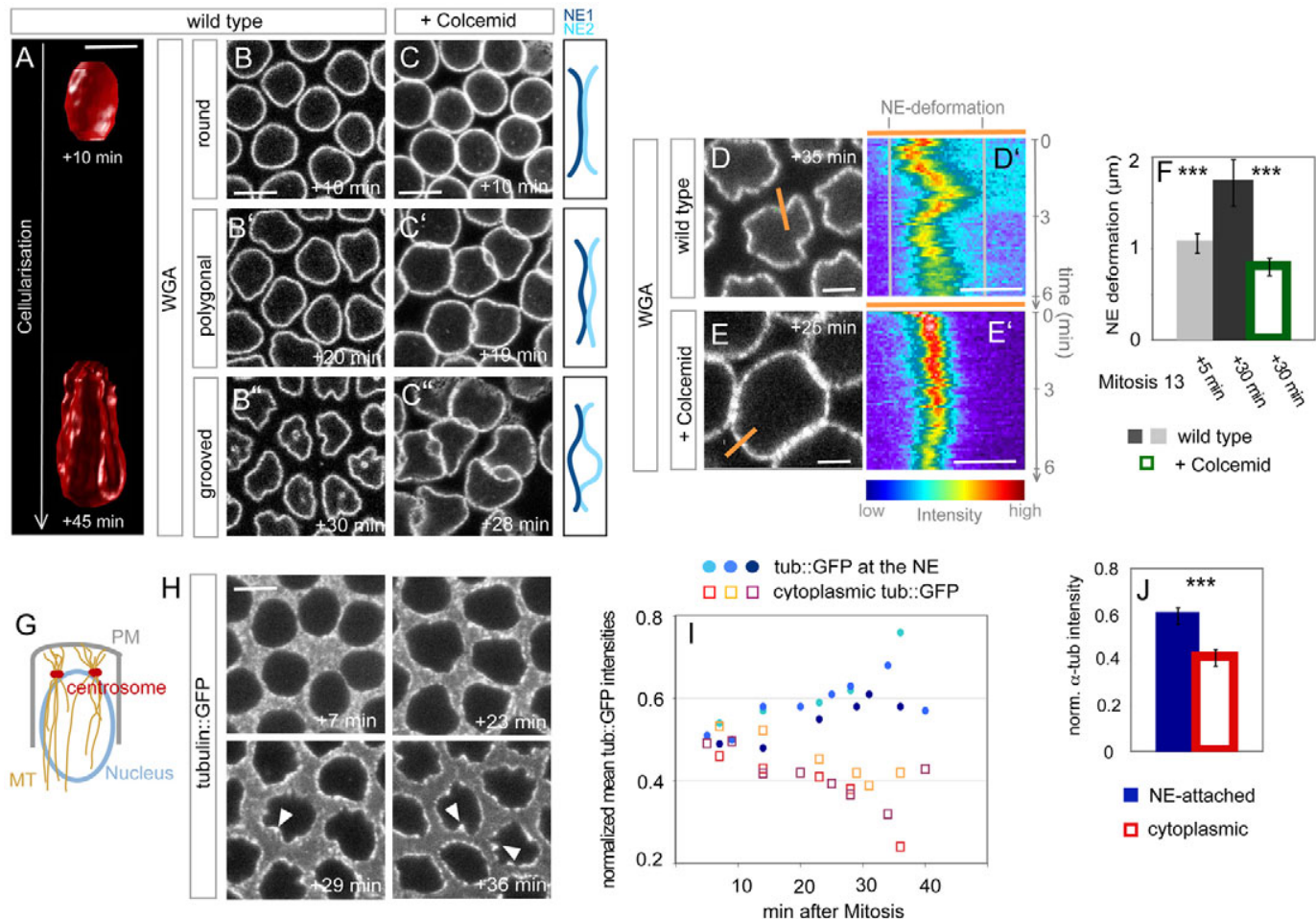

Fig. 1. Microtubules (MTs) control nuclear envelope (NE) morphogenesis during Drosophila cellularization. (A-C) MTs regulate NE shape. (A) 3D reconstruction from serial $z$-sections of a nucleus from a wild-type embryo imaged 10 or 45 minutes after start of interphase 14. Scale bar: $5 \mu \mathrm{m}$. (B-B") Top-view stills from time-lapse movies of a wild-type embryo injected with WGA-Alexa488 showing round (B), polygonal ( $\left.B^{\prime}\right)$ or grooved $\left(B^{\prime \prime}\right)$ nuclei. Indicated times are relative to the start of interphase 14. Scale bar: $5 \mu \mathrm{m}$. (C-C') Top-view stills of a wild-type embryo injected with Colcemid and WGA-Alexa488. Indicated times are relative to the start of interphase 14. Scale bar: $5 \mu$ m. Schematics depict NE arrangements. (D-F) NE dynamics and groove formation depend on MTs. NE deformations are measured as the distance between the most lateral positions of the signal from WGA-Alexa488 kymographs $\left(D^{\prime}, E^{\prime}\right)$ from time-lapse movies of wild-type $\left(D, D^{\prime}\right)$ or Colcemid-injected $\left(E, E^{\prime}\right)$ embryos recorded for 6 minutes. One respective frame is shown in $D, E$, where the position of the kymograph is indicated by the orange bar. Times refer to the start of interphase 14. Scale bars: $2 \mu \mathrm{m}$ in $D, E ; 1 \mu \mathrm{m}$ in $D^{\prime}, E^{\prime}$. Note the reversibility of groove formation ( $\left.D^{\prime}\right)$. (F) NE fluctuations are different in nuclei from either younger $(n=9)$ and older $(n=14)$ cellularizing embryos, or between wild-type and Colcemid-treated embryos $(n=8)$, respectively. $* * * P<0.001$. (G-I) MTs accumulate at the NE during cellularization. (G) Schematic representation of MT organization during cellularization in a sagittal view. PM, plasma membrane. (H) Top-view stills from a time-lapse movie of a tub::GFP-expressing embryo during cellularization. Indicated times are relative to start of interphase 14. Note the pronounced accumulation of tub::GFP in grooves (arrowheads). Scale bar: $5 \mu$ m. (I) Quantification of normalized tub::GFP at the NE or in the cytoplasm for three embryos. Plotted are mean intensities of six nuclei for each embryo. (J) Quantification of normalized $\alpha$-Tubulin levels at the NE or in the cytoplasm based on antibody stainings on fixed wild-type embryos in late cellularization $\left(P=7.29 \times 10^{-4}, n=4\right.$ embryos, 44 nuclei, 53 cytoplasmic regions). ${ }^{*} * P<0.001$. 

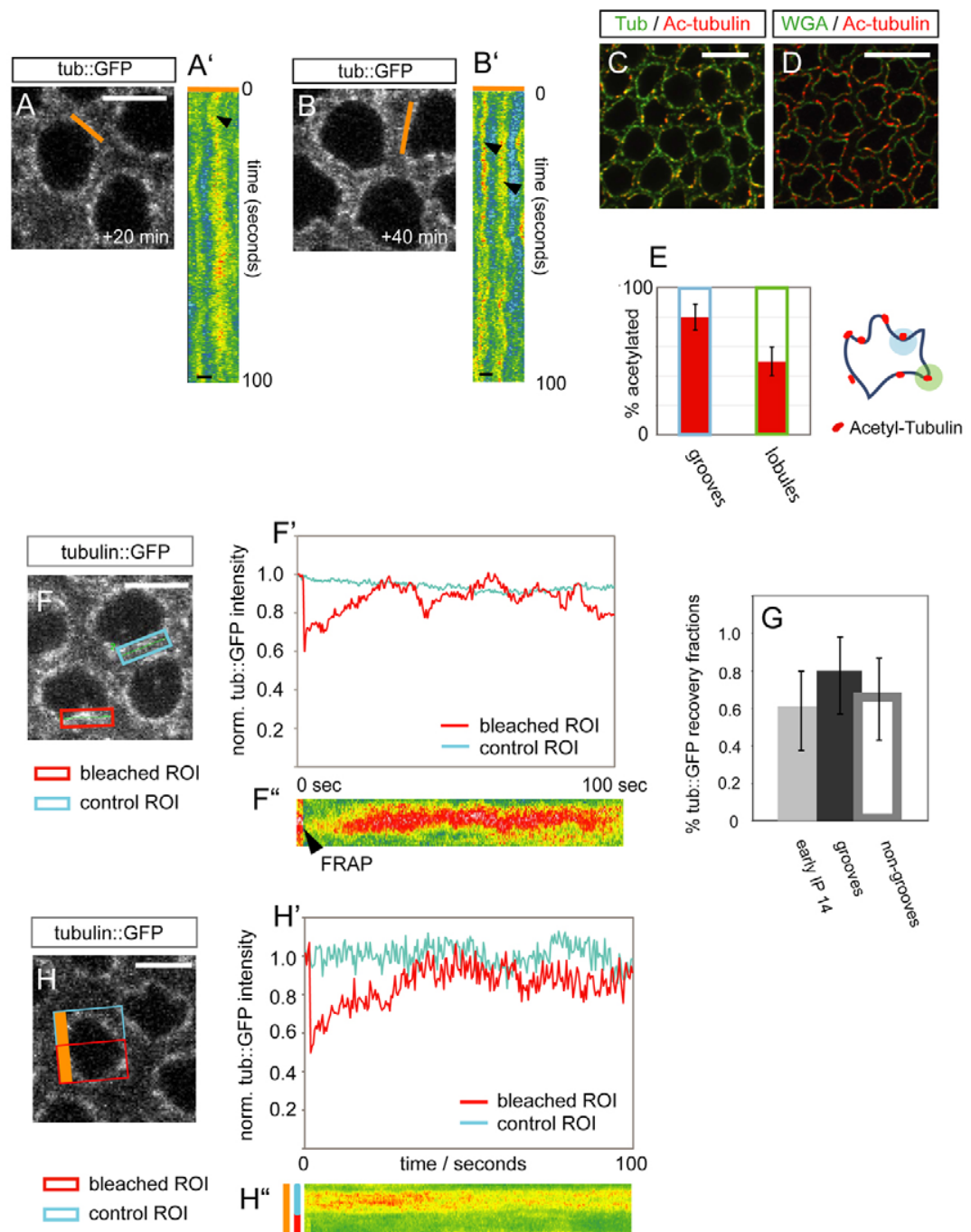

Fig. 2. Dynamic microtubules (MTs) are organized in stationary bundles at the Drosophila nuclear envelope (NE). (A,B) MTs occur in bundles along the NE. Top-view stills $(A, B)$ and kymographs $\left(A^{\prime}, B^{\prime}\right)$ from timelapse movies of tub::GFP-expressing embryos, before $\left(A, A^{\prime}\right)$ or after $\left(B, B^{\prime}\right)$ groove formation. Scale bars: $5 \mu \mathrm{m}$ in $A ; 1 \mu \mathrm{m}$ in $A^{\prime}, B^{\prime}$. MT bundles are indicated by arrowheads $\left(A^{\prime}, B^{\prime}\right)$. Note that bundles keep their lateral position. (C-E) A subset of MTs along the NE is acetylated and decorates grooves. Top views from fixed wild-type embryos during cellularization (C,D). Acetylated Tubulin marks a subset of the total MT population (C) which is distributed along the NE (D). Scale bars: $10 \mu \mathrm{m}$.

(E) Quantification of the percentage of grooves and lobules, which are occupied by an acetylated Tubulin spot, as counted from images such as D. Error bars represent s.d. (F,G) MTs within bundles are dynamic and grow in an apical-basal direction. (F-F") Top-view still of a cellularizing embryo expressing tub::GFP prior to photobleaching (F). Scale bar: $5 \mu \mathrm{m}$. The red ROI was bleached and recovery is shown in $\mathrm{F}^{\prime}$. Tub::GFP recovery occurs at the site of the bundle prior to bleaching $\left(\mathrm{F}^{\prime \prime}\right)$. (G) Recovery fractions of tub::GFP after FRAP are similar between embryos in early ( $n=7$ nuclei) or late cellularization and between grooves $(n=5)$ and non grooves $(n=8)$ respectively. Error bars represent s.d. $\left(\mathbf{H}-\mathbf{H}^{\prime \prime}\right)$ Top-view still of a cellularizing embryo expressing tub::GFP $(H)$. Scale bar: $5 \mu \mathrm{m}$. The red half of the nucleus was bleached and recovery of the tub::GFP signal is shown in $\mathrm{H}^{\prime}$. Note that intensity of the control (blue) half of the nucleus does not decrease $\left(\mathrm{H}^{\prime}\right)$, indicating MT recovery from apical basal growth and not from lateral sliding. Accordingly a kymograph $\left(\mathrm{H}^{\prime \prime}\right)$ spanning bleached and control regions (indicated with the orange bar in $\mathrm{H}$ ) shows no lateral migration of the tub::GFP signal from the control to the bleached region. supplementary material). Thus, groove formation did not correlate in time with total NE surface increase. Kymographs also showed that grooves could be reversible within a few minutes, suggesting that active mechanisms might underlie their formation (see Movie 1 in the supplementary material; Fig. 1D'). We next tested whether NE lobulations form in response to active stress exerted by the invaginating membrane and contractile furrow canals as they pass around nuclei. We imaged the NE in slam RNAi embryos in which plasma membrane invagination is strongly delayed up until the end of nuclear elongation (Lecuit et al., 2002). In these embryos, groove formation was indistinguishable from wild type (see Fig. $\mathrm{S} 1 \mathrm{~B}, \mathrm{~B}^{\prime}$ in the supplementary material).

This led us to hypothesize that groove formation is an active process operating specifically at the NE, as already suggested in cell culture (Olins and Olins, 2004). During cellularization, MTs form a tight basket around nuclei. They are organized from an apical pair of centrosomes (Fig. 1G) and are known to be required for nuclear elongation (Schejter and Wieschaus, 1993). We investigated the relationship between MT distribution and NE grooves. We imaged embryos expressing tubulin::GFP (see Movie 2 in the supplementary material; Fig. $1 \mathrm{H}$ ) and measured a gradual concentration of tub::GFP at the NE (Fig. 1I). Similar observations were made with endogenous $\alpha$-Tubulin labeled with an antibody
(Fig. 1J). Remarkably, MTs consistently decorated grooves (see Movie 2 in the supplementary material; Fig. 1H). To test whether MTs could control groove formation, we injected the MTdepolymerizing drug Colcemid during early cellularization. As previously reported, elongation of nuclei was blocked (Edgar et al., 1987) but NE surface continued to grow, although at a reduced rate (see Fig. S2A in the supplementary material). Kymographs indicated that the dynamics of the NE was drastically impaired compared with wild type $\left(P=1.34 \times 10^{-4}\right)$ (see Movie 3 in the supplementary material; Fig. 1E,F) and reversible grooves never formed. However, live imaging revealed the existence of irreversible buckling, when NE growth brings nuclei into contact. In this case, deformations form passively in response to the compressive stress imposed by NE growth (see Movie 3 in the supplementary material; Fig. $1 \mathrm{C}-\mathrm{C}^{\prime \prime}$ ). We conclude that MTs are concentrated in grooves and are essential for NE remodeling.

\section{Dynamic microtubules are organized in stationary bundles at the NE}

The observations described above suggest that MTs might constrain the NE and deform it locally like static posts. Consistent with this, kymograph analysis from embryos expressing tub::GFP indicated that MTs were organized within stationary bundles that 
kept their lateral localization along the NE (Fig. 2A,B). Bundles were present during early (Fig. 2A, $\mathrm{A}^{\prime}$ ) and late cellularization (Fig. $\left.2 \mathrm{~B}, \mathrm{~B}^{\prime}\right)$. Acetylated, and thus relatively stable, MTs have been reported to localize at the NE during cellularization but not in earlier interphases (Wolf et al., 1988). This acetylated pool represented a subset of the overall MT network around nuclei (Fig. $2 \mathrm{C})$. Most grooves $(79.8 \pm 0.09 \%, n=190)$ were occupied by acetylated MTs. Nonetheless, MT bundles were also found outside grooves as half of the NE lobules $(49.8 \pm 0.1 \%, n=407)$ contained acetylated MT spots (Fig. 2D,E). Together, this supports the view that MT bundles behave like stable posts constraining the NE. To test this further, we probed MT dynamics by performing fluorescence recovery after photobleaching (FRAP) experiments in embryos expressing tub::GFP. Strikingly, however, MTs within bundles appeared unexpectedly dynamic (recovery fraction $=64 \pm 21 \%, t_{1 / 2}=6.8 \pm 5.1$ seconds) (Fig. 2F,G,H). Tub::GFP recovered at the same position along the $\mathrm{NE}$ as where the bundle was located before bleaching (Fig. 2F") and we did not observe lateral redistribution of MTs from adjacent regions of the NE (Fig. $\left.2 \mathrm{H}, \mathrm{H}^{\prime}, \mathrm{H}^{\prime \prime}\right)$. This indicates that MTs are inherently dynamic within apicobasally oriented stationary bundles and do not slide laterally along the NE. MTs were equally dynamic before and after nuclei lost their regular structure $(P=0.275)$ and, in late cellularizing embryos, grew similarly in grooves and lobules $(P=0.354)$ (Fig. $2 \mathrm{G})$. Imaging a GFP-fusion of the MT plus end tracking protein EB1 confirmed the dynamic nature of the MT network along the NE (see Fig. S3A in the supplementary material).

\section{NE fluctuations require dynamic microtubules}

We, therefore, considered another model where MT polymerization might play an active role in NE deformations. Closer examination of NE dynamics revealed two modes of fluctuations that could be deciphered from kymographs: apart from the large $(1.7 \pm 0.2 \mu \mathrm{m})$ and few-minutes long deformations associated with grooves (Fig. $1 D^{\prime}$, Fig. 3A), the NE showed 'high-frequency' fluctuations with a lower amplitude of up to $300 \mathrm{~nm}$ (Fig. 3A'). Interestingly, MT depolymerization caused a significant reduction in the amplitude of the latter fluctuations $\left(P=6.25 \times 10^{-28}\right)$, indicating that those probably result from impulsions associated with MT polymerization events (Fig. 3B). Consistent with this, NE fluctuations were also low in interphase 13 embryos $\left(P=3.73 \times 10^{-14}\right.$ compared with interphase 14$)$, when MTs are not concentrated at the NE (Fig. 3B). To test this further, we injected the MT-stabilizing drug Taxol. This decreased MT dynamics, as EB1::GFP was reduced at the NE (see Fig. S3A, $A^{\prime}$ in the supplementary material) and MTs recovered less and more slowly (recovery fraction $=46 \pm 16 \%, t_{1 / 2}=22.0 \pm 6.8$ seconds) compared with wild type after photobleaching (see Fig. S3B in the supplementary material). Taxol injection significantly reduced the high-frequency fluctuations of the NE $\left(P=1.66 \times 10^{-7}\right)$ (Fig. 3C). Taxol prevents incorporation of tubulin monomers, but also stabilizes existing tubules. Consequently, the lifetime of MT bundles at the NE was increased following Taxol injection (see Fig. S3C, $C^{\prime}, \mathrm{D}$ in the supplementary material) $(P=0.0013)$, whereas the number of bundles was similar to controls $(P=0.319)$ (data not shown). Although MT bundles became more stable, the NE was more regular and the formation of grooves was strongly impaired after Taxol injection (Fig. 3D-F). We conclude that the NE is a very dynamic organelle exhibiting two modes of fluctuations that are both dependent on MT dynamics. Moreover, grooves emerge from the collective effects of MT polymerization events concentrated in stationary (but not stable) bundles at the NE.
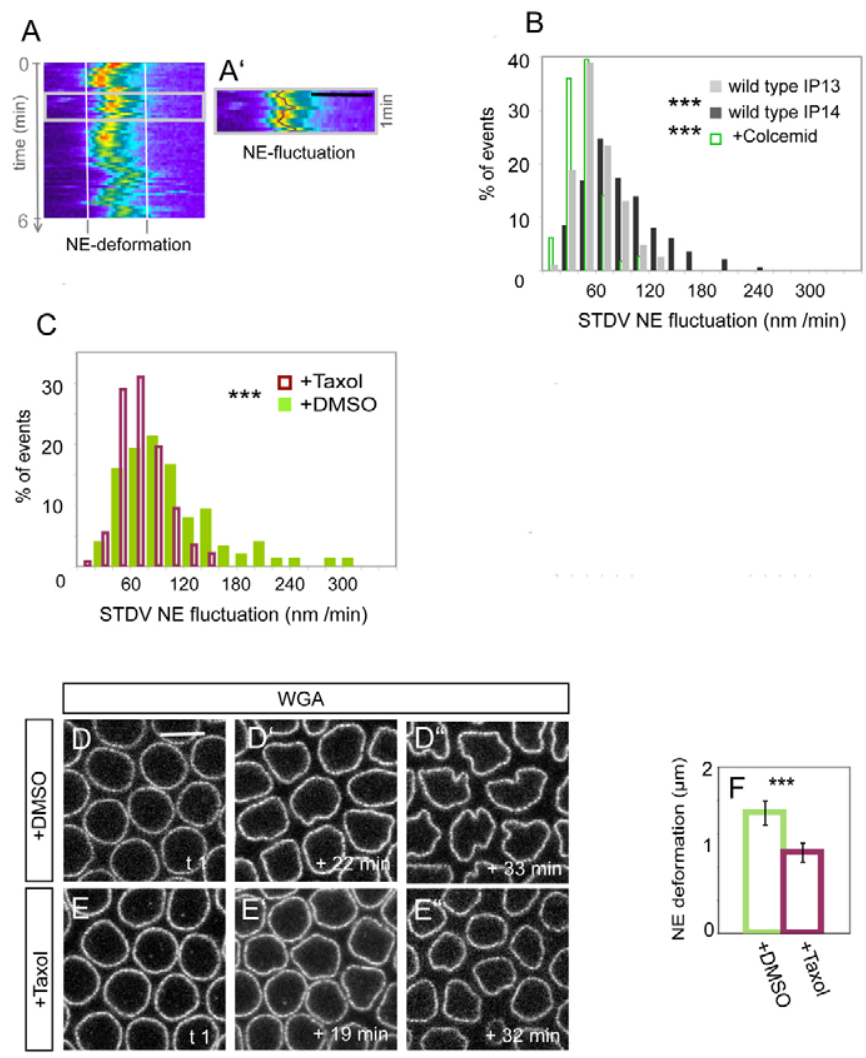

Fig. 3. Dynamic microtubules (MTs) trigger nuclear morphogenesis in Drosophila. $\left(\mathbf{A}, \mathbf{A}^{\prime}\right)$ Two modes characterize nuclear envelope (NE) dynamics. Besides the large-scale deformations indicated by the distance between the most lateral positions in the NE kymograph over 6 minutes (A), high-frequency NE fluctuations are measured as standard deviations (STDV) of the lateral positions of the intensity maxima from WGA-Alexa488 kymographs (indicated by the black line following the signal in $A^{\prime}$ ) recorded every 5 seconds over 1minute intervals. Scale bar: $1 \mu \mathrm{m}$ in $\mathrm{A}^{\prime}$. (B) NE fluctuations start with cellularization and depend on MTs. Histogram of STDV from NE fluctuations for wild-type embryos in interphase 14 ( $n=204$ 1-minute intervals) or interphase 13 ( $n=135$ ) or from Colcemid-injected cellularizing embryos ( $n=114)$. (C) Impaired MT dynamics reduces highfrequency fluctuations of the NE. Histogram of the STDV from highfrequency NE fluctuations in DMSO- (n=150 1-minute intervals) or Taxol-injected ( $n=149$ ) cellularizing embryos. (D-F) Blocking MT dynamics impairs NE groove formation. Top-view stills (D-E") from embryos co-injected with WGA-Alexa488 and either DMSO (D- $\left.D^{\prime \prime}\right)$ or Taxol $\left(E-E^{\prime \prime}\right)$. Indicated time points are relative to t1 in D,E. Scale bar: $5 \mu \mathrm{m}$. (F) Large-scale NE deformations are reduced in Taxol-injected embryos $(n=9)$ compared with controls $(n=10)\left(P=1.57 \times 10^{-4}\right)$. $* * * P<0.001$. Error bars represent s.d.

\section{Cytoplasmic Dynein bundles microtubules at the NE}

This model predicts that affecting MT bundling should also affect NE lobulation. Dynein might affect MT concentration at the NE, e.g. by regulating bundle organization. Dynein connects MTs to cargo molecules or organelles and regulates centrosome attachment at the NE in Caenorhabditis elegans (Gonczy et al., 1999) and Drosophila (Robinson et al., 1999). To interfere specifically with Dynein activity during cellularization, we injected anti-Dynein heavy chain (Dhc) or anti-Dynein intermediate chain (Dic) neutralizing antibodies into embryos after they entered interphase 
14. This impaired nuclear elongation, resembling a weak Colcemid phenotype (see Fig. S2B in the supplementary material). To check MT integrity in embryos with reduced Dynein activity, we coinjected neutralizing antibodies and WGA-Alexa555 into cellularizing embryos expressing tub::GFP and compared tub::GFP levels at the NE within the same embryos inside and outside the injection area (Fig. 4A-C). Ratios between NE-decorating and cytoplasmic tub::GFP were significantly reduced in regions where the neutralizing anti-Dynein antibodies had been injected, compared with adjacent regions (Fig. 4C). Similar effects were observed for anti-Dhc ( $n=20$ nuclei, $\left.P=1.28 \times 10^{-7}\right)$ and anti-Dic antibodies $\left(n=50\right.$ nuclei, $\left.P=2.29 \times 10^{-8}\right)$ but not with anti-GST antibodies ( $n=50$ nuclei, $P=0.79$ ) (Fig. 4C). To test if this is due to reduced MT polymerization (Han et al., 2001), we quantified EB1::GFP levels at the NE and found no significant difference between anti-Dhc antibody-targeted or untargeted areas within the same embryos (see Fig. S4A,B in the supplementary material). Consequently, MT polymerization-dependent fluctuations of the NE were similar in wild-type embryos or after Dynein inhibition $(P=0.563)$ (see Fig. S4C in the supplementary material). Instead, tub::GFP kymographs showed that bundles were shorter lived and splayed out following anti-Dhc antibody injection, in contrast to control regions where bundles were persistent $\left(P=5.65 \times 10^{-34}\right)$ (Fig. 4D,E). We conclude that Dynein is required for MT bundle integrity at the NE. Remarkably, in contrast to a control anti-GST antibody, injection with either anti-Dhc or anti-Dic antibodies reduced significantly the formation of grooves (Fig. 4F-I). Together, this suggests that MT dynamics is responsible for highfrequency fluctuations of the NE and bundling of dynamic MTs is a key determinant of large-scale NE deformations into grooves through the collective effect of polymerization forces. Dynein could also control NE deformations in grooves by coupling to the NE directly and using MT bundles as a (dynamic) scaffold.

As in other systems, Dynein could be anchored at the NE by the LINC (linker of nucleoskeleton and cytoskeleton) complex. The LINC complex consists of a KASH domain protein and a SUN domain protein, and spans the NE. Double mutants for the only two Drosophila KASH domain proteins Msp-300 and Klarsicht were reported previously to have no defects in nuclear morphology or position in early embryogenesis (Technau and Roth, 2008) Flies encode two SUN domain proteins. Only one of them is expressed in embryos and is encoded by two currently independently annotated genes CG3287 and klaroid (Technau and Roth, 2008), while the other SUN domain protein, Spag4 (Sperm-associated antigen 4, previously known as as Giacomo) is expressed specifically in testis (Kracklauer et al., 2010). We thus looked at embryos from parents homozygous for the hypomorphic allele $P\{E Y 2 g\} C G 3287^{E Y 03560}$, which phenocopies the rough eye phenotype of a deletion comprising both CG3287 and klaroid (see Fig. S5A-A" in the supplementary material) (Technau and Roth, 2008). In contrast to Dynein inhibition, $P\{E Y 2 g\} C G 3287^{E Y 03560}$ embryos exhibited normal groove formation (data not shown). Yet, NE fluctuations were enhanced in $P\{E Y 2 g\} C G 3287^{E Y 03560}$ embryos (see Fig. S5B in the supplementary material) $\left(P=5.4 \times 10^{-7}\right)$, consistent with the proposed role of the LINC complex to buffer/dampen cytoskeletal stresses at the NE (King et al., 2008).

\section{The farnesylated protein Kuk mediates MT- induced nuclear deformations}

How do MT forces interplay with the material properties of the NE to yield effective deformations? The farnesylated inner NE protein Kuk is essential for NE lobulation during cellularization (Fig. 5A-
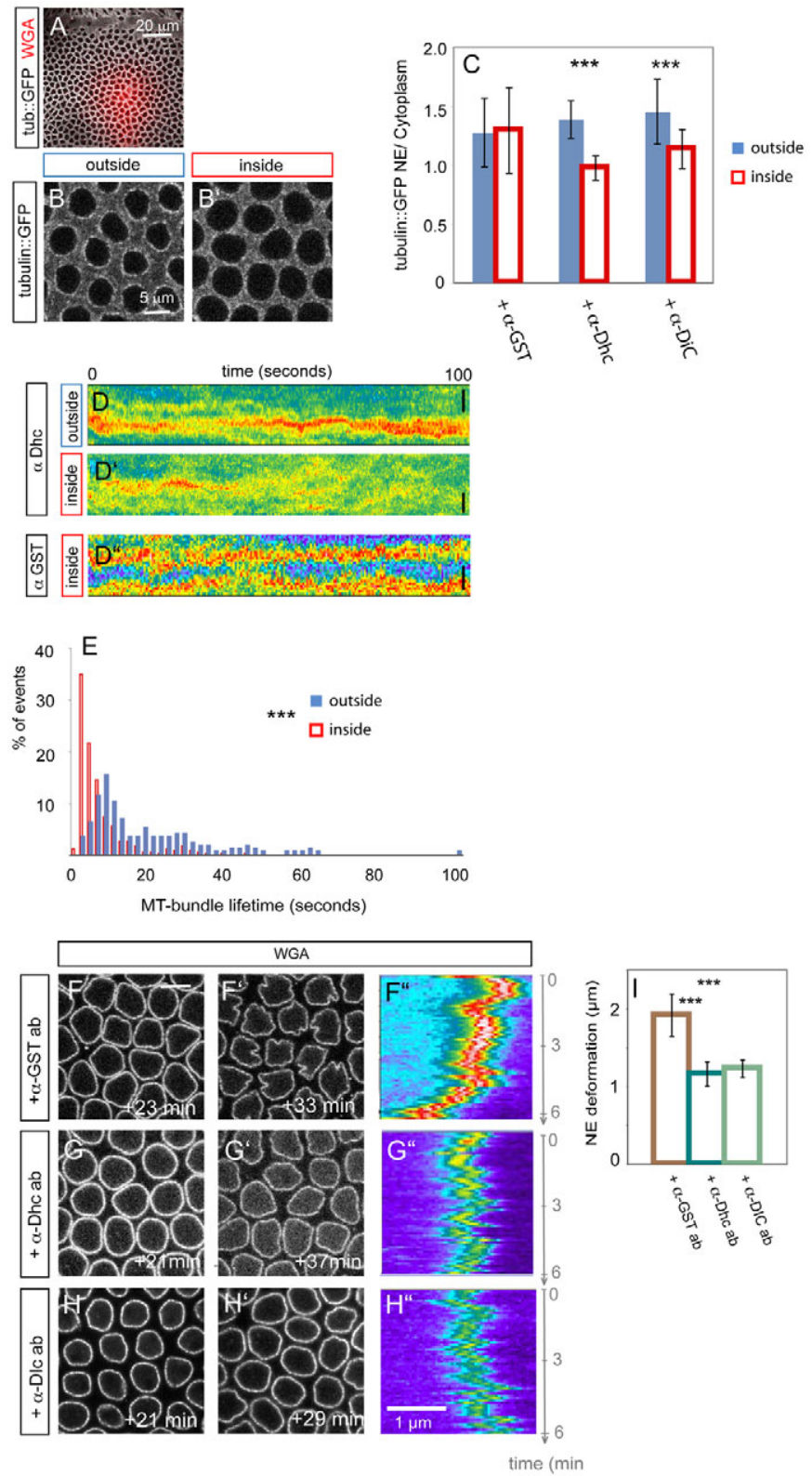

Fig. 4. Cytoplasmic Dynein bundles microtubules (MTs) at the Drosophila nuclear envelope (NE). (A-C) MTs are reduced at the NE upon Dynein inactivation. Top-view stills from a movie recording a tub::GFP-expressing embryo co-injected with $\alpha$-Dhc antibody and WGAAlexa555 $(A, B)$. Outside-inside distinction is based on the presence of WGA-Alexa555 (A). (C) Quantification of tub::GFP levels around the NE and in the cytoplasm. Plotted are the ratios of NE attached/cytoplasmic tub::GFP outside and inside the injection area after injection with the respective antibodies. ${ }^{*} * P<0.001$. Error bars represent s.d. (D,E) Dynein is required for the integrity of MT bundles at the NE. Tub::GFP kymographs $\left(D-D^{\prime \prime}\right)$ recorded over 100 seconds from either $\alpha$-Dhc $\left(D, D^{\prime}\right)$ or $\alpha-G S T\left(D^{\prime \prime}\right)$ antibody-injected embryos. Scale bars: $1 \mu \mathrm{m}$. (E) Histogram of MT bundle lifetimes at the NE inside ( $n=388$ bundles) or outside ( $n=177$ bundles) the $\alpha$-Dhc antibody targeted region, as measured from kymographs such as that shown in $D, D^{\prime}$. (F-I) Dynein inhibition affects nuclear morphogenesis. Top-view stills and kymographs of the NE from either $\alpha-G S T\left(F-F^{\prime \prime}\right), \alpha-D h c$ $\left(\mathrm{G}-\mathrm{G}^{\prime \prime}\right)$ or $\alpha-\mathrm{DIC}\left(\mathrm{H}-\mathrm{H}^{\prime \prime}\right)$ antibody-injected embryos. The indicated times are relative to the start of interphase 14 . Scale bar: $5 \mu \mathrm{m}$. (I) Comparison of large-scale NE deformations about 30 minutes after start of interphase 14 between $\alpha$-GST $(n=9), \alpha$-Dhc $(n=10)$ or $\alpha$-DIC $(n=15)$ antibody-injected embryos ( $\alpha$-GST/ $\alpha$-Dhc: $P=2.39 \times 10^{-4} ; \alpha-$ GST/ $\alpha$-DIC: $\left.P=5.7 \times 10^{-5}\right)$.

$\star * * P<0.001$. Error bars represent s.d. 

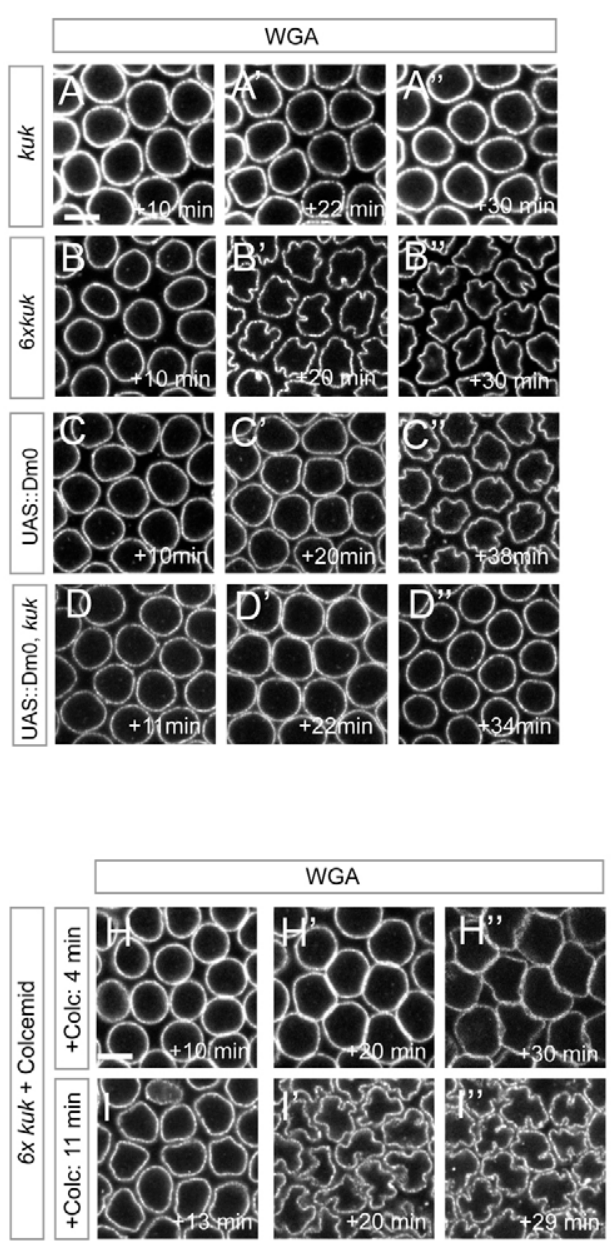

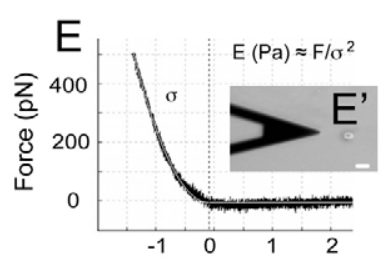

tip sample separation ( $\mu \mathrm{m})$
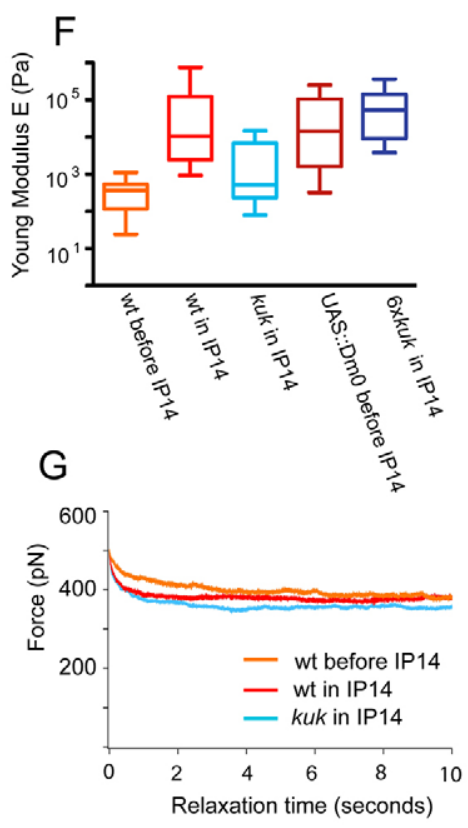

Fig. 5. The farnesylated protein Kuk mediates microtubule (MT)-induced nuclear deformation in Drosophila. (A-D") Farnesylated inner nuclear membrane (INM) proteins and nuclear morphology. Top-view stills taken at one medial z-plane from embryos of the respective genotypes injected with WGA-Alexa488. Indicated times are relative to entry into interphase 14. $(A, B)$ Kuk is required for nuclear envelope (NE) deformation ( $\left.A-A^{\prime \prime}\right)$, and its overexpression leads to stronger and premature $\mathrm{NE}$ lobulation (B-B"). (C-D") Dm0 overexpression does not phenocopy 6xkuk expression (C-C") and cannot rescue the lobulation defects in kuk mutants (D-D").

(E-G) Farnesylated INM proteins regulate NE stiffness. (E) Typical pressing force curve with a Hertz fit for a pyramidal intender (white line) for an isolated nucleus. For a given pressing force $F$ the Young's modulus $E$ is inversely proportional to the square of the indentation $\sigma$. (E') Photomicrograph of an AFM cantilever close to a single immobilized nucleus. Scale bar: $20 \mu \mathrm{m}$. (F) Whisker plots of E moduli measured for nuclei isolated from embryos of the indicated genotypes and developmental stages. (G) Average force dissipation curves ( $F$ versus $t$ ) after pressing on isolated nuclei over 10 seconds are similar between nuclei from wild-type embryos during ( $n=13$ nuclei) or before interphase $14(n=11)$ or from kuk mutants $(n=11)$. $\left(\mathbf{H}-\mathbf{I}^{\prime \prime}\right)$ Kuk stabilizes MT-induced NE deformations. (H,I) Top-view stills from $6 x k u k$ embryos after Colcemid injection. Colcemid treatment shortly after mitosis $\left(\mathrm{H}-\mathrm{H}^{\prime \prime}\right)$ abolishes MTinduced NE deformation and shows buckling due to lateral nuclear growth. MT depolymerization later in cellularization allows NE deformation $\left(I^{\prime}, I^{\prime \prime}\right)$. All indicated time points are relative to the start of interphase 14. Scale bars: $5 \mu \mathrm{m}$ in $\mathrm{A}, \mathrm{H}$.
$\mathrm{A}^{\prime \prime}$ ) (Brandt et al., 2006; Pilot et al., 2006). In kuk mutants the NE displayed high-frequency oscillations similar to wild type (see Fig. $\mathrm{S} 6 \mathrm{C}$ in the supplementary material) but eventually failed to deform into grooves (Fig. 5A-A"; see Movie 4 in the supplementary material). Consistent with this, neither MT attachment at the NE (Pilot et al., 2006) nor MT dynamics or organization into bundles was changed in cellularizing kuk embryos (see Fig. S6A,B in the supplementary material). Considering its localization at the inner nuclear membrane, this argues that Kuk affects nuclear deformations by exclusively modulating NE material properties essential for NE deformations.

The visco-elastic properties of the NE could indeed control its deformability. We tested this with atomic force microscopy (AFM), which has been successfully used to probe the mechanical characteristics of cells and isolated nuclei (Lammerding et al., 2007). The application of a given force by the AFM cantilever tip indents a single nucleus. From the resulting pressing curve the material's stiffness expressed by the Young's modulus E can be obtained with a suitable Hertz model (Fig. 5E, E'). We first probed immobilized nuclei that were isolated from wild-type embryos either before (blastoderm stage, $n=15$ nuclei) or during ( $n=20$ nuclei) interphase 14 and obtained Young's moduli in a similar range to that published for other systems (Dahl et al., 2008). Interestingly, nuclei from embryos before interphase 14 appeared soft with $\mathrm{E}$ moduli far below the values obtained from interphase 14 embryos $\left(P=4.74 \times 10^{-8}\right)$ (Fig. 5F), indicating that stiffness rises as grooves form. Strikingly, nuclei from kuk mutant interphase 14 embryos ( $n=46$ nuclei) showed much lower E moduli than wildtype nuclei of the same age $\left(P=5.07 \times 10^{-9}\right)$ (Fig. 5F). We conclude that Kuk induces stiffness of the NE during cellularization.

However, several lines of evidence indicate that Kuk controls additional properties of the NE under stress. Overexpression of $k u k$ induces stronger and precocious $\mathrm{NE}$ deformations during cellularization (Brandt et al., 2006) (Fig. 5B-B"). Importantly, this depends on the presence of MTs, in agreement with our model that sustained dynamic MTs impose the active stress required for NE morphogenesis (Fig. 5H- $\mathrm{H}^{\prime \prime}$ ). However, nuclei from kukoverexpressing embryos were not stiffer than wild-type nuclei $(P=0.491)$ (Fig. 5F). Nuclei from embryos with elevated levels of the Drosophila B type Lamin Dm0 (Lam - FlyBase) showed a significantly increased stiffness prior to interphase 14 compared with nuclei from wild-type embryos $\left(P=4.95 \times 10^{-5}\right)$ (Fig. 5F), but did not deform the $\mathrm{NE}$ as strongly as in 6xkuk embryos during cellularization (Fig. 5C-C"). Finally, increased levels of Dm0 could not rescue $k u k$ mutants (Fig. 5D-D") (Polychronidou et al., 2010). Overall this argues against a model in which Kuk regulates deformation of the NE solely by increasing its stiffness.

We thus tested whether Kuk could modulate nuclear viscosity by comparing force dissipation after pressing on isolated nuclei with the AFM cantilever. However, recording force curves over 10 seconds did not reveal significant differences between wild-type nuclei from embryos before and during cellularization or between the latter and nuclei isolated from kuk embryos in interphase 14 (Fig. 5G). 
This led us to propose that Kuk could act structurally to stabilize NE deformations initially induced by progressive MT stress. Once stabilized, the NE could stay deformed without any further need of a cytoplasmic force. To test this, we depolymerized MTs later in cellularization, once nuclei have lost their round shape. However, at this stage the invaginated plasma membrane restricted MT depolymerization basally to the cellularization front (see Fig. S7 in the supplementary material). To overcome this, we depolymerized MTs in 6xkuk, which acquire NE deformations earlier than do wild type (Fig. 5B-B"). Colcemid application early after mitosis prevented any $\mathrm{NE}$ deformation and resembled similarly treated embryos with wildtype levels of Kuk (Fig. 5H-H") By contrast, later MT depolymerization once nuclei had lost their spherical shape allowed groove formation (Fig. 5I-I"). This proposes a two-tiered mechanism for NE deformation during Drosophila cellularization. Sustained MT polymerization forces in bundles first nucleate deformations of the NE. Subsequently, these deformations are enhanced into grooves by the activity of Kuk at the INM, which causes NE stiffening and buckling in response to stress.

\section{NE dynamics induces chromatin mobility}

Finally, we tested whether NE dynamics and deformation could be transmitted to the nucleoplasm and thereby globally 'agitate' the chromatin. This could contribute to the progressive activation of the chromatin and to zygotic activation occurring during cellularization (Pilot et al., 2006; Rudolph et al., 2007).
We imaged a subset of the chromatin labeled by a GFP fusion of the Histone variant $\mathrm{H} 2 \mathrm{Av}$ (His2Av - FlyBase) during cellularization (Clarkson and Saint, 1999). H2Av::GFP was localized throughout nuclei and was abundant in spots, which were very dynamic (Fig. 6A, $\mathrm{A}^{\prime}$; see Movie 5 in the supplementary material). Spot dynamics was not restricted to the nuclear periphery but occurred also in the central nucleoplasm. There, spots moved relative to each other (Fig. 6B) and occasionally followed NE movements (Fig. 6A'). We measured mean velocities of H2Av::GFP by applying particle image velocimetry (PIV) to quantitate flow fields (Fig. 6B-C'). The mean velocity was commensurate with that of the NE and increased from interphase 13 to 14 (Fig. 6C), concomitant with the emergence of NE fluctuations (Fig. 3B). Strikingly, H2Av::GFP mean velocities decreased after Colcemid injection (Fig. 6C; see Movie 6 in the supplementary material). Similar results were obtained when the PIV analysis was performed considering only the central region of the nucleoplasm (Fig. 6C'), thereby reflecting global effects on the chromatin. In kuk mutants, H2Av::GFP flow mean velocities were also significantly reduced, albeit less than after Colcemid treatment (Fig. 6C,C'; see Movie 7 in the supplementary material). As PIV does not follow individual chromatin spots, we tracked bright H2Av::GFP spots that could be resolved in kymographs in sections through the nucleoplasm (Fig. 6D-G). In a given confocal plane, the length of an H2Av::GFP trace in the nucleoplasm measures the persistence of $\mathrm{H} 2 \mathrm{Av}:$ :GFP spots in this plane. Kymograph analysis in subsequent $z$-planes indicated that H2Av::GFP spots predominantly moved along the apical basal axis (see Fig. S8 in the
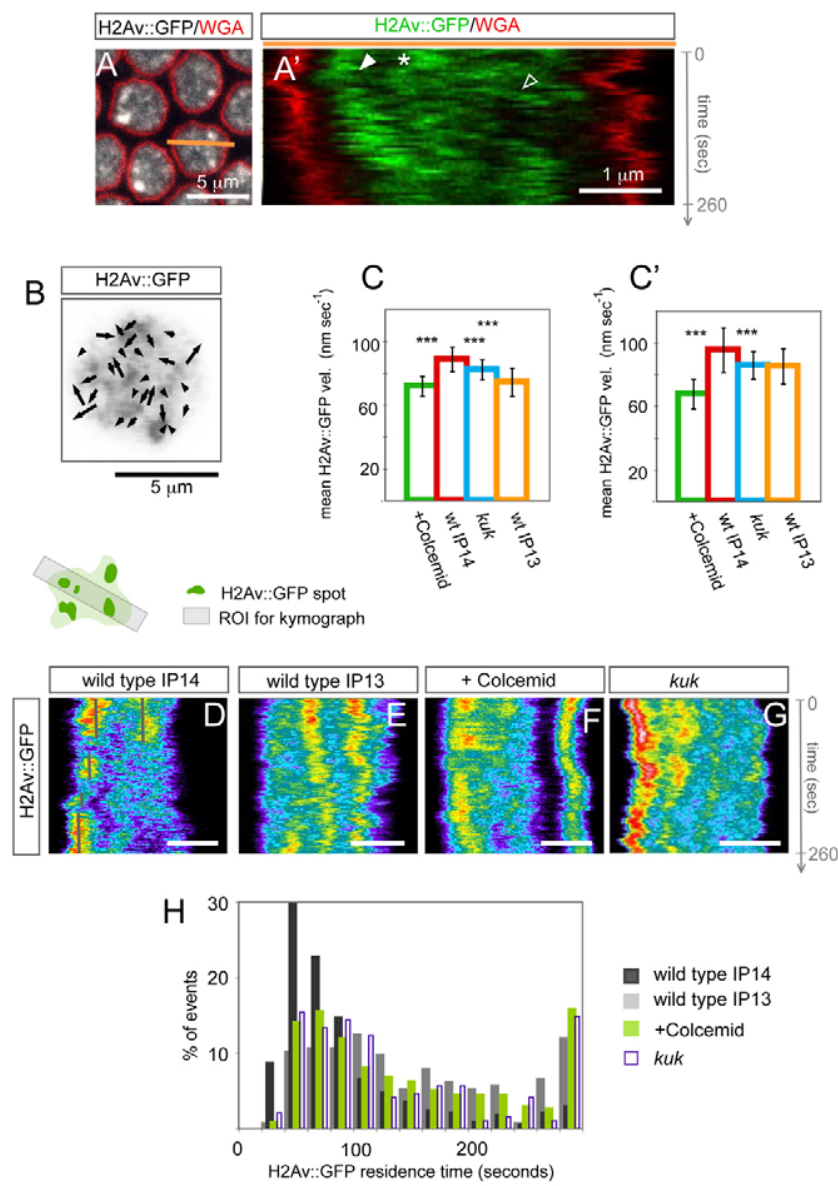

Fig. 6. Nuclear envelope (NE) dynamics induces chromatin mobility. Chromatin is dynamic in cellularizing Drosophila embryos. (A, $\left.\mathbf{A}^{\prime}\right)$ Top-view still $(A)$ and kymograph $\left(A^{\prime}\right)$ from a time-lapse movie imaging one $z$-plane through nuclei of a cellularizing embryo expressing H2Av::GFP injected with WGA-Alexa555. H2Av::GFP is diffuse throughout nuclei and concentrates in spots which can be tracked in kymographs $\left(A^{\prime}\right)$. The region depicted in the kymograph corresponds to the orange bar in $A$. $\left(A^{\prime}\right)$ H2Av::GFP spots move parallel to NE deformations adjacent to the NE (solid arrowheads) but also in the nuclear interior (open arrowhead), or move independently of the NE track (asterisk). (B-C') Analysis of chromatin dynamics by particle image velocimetry (PIV). (B) Nucleus from a H2Av::GFP-expressing interphase 14 control embryo with the corresponding vector field for two subsequent frames. The random orientation of vectors indicates $\mathrm{H} 2 \mathrm{Av}$ :: GFP flows relative to each other. The H2Av:: GFP signal is inverse colored. $\left(C, C^{\prime}\right)$ Chromatin velocities are decreased when NE dynamics are reduced or morphogenesis is inhibited. (C) Mean H2Av::GFP velocities calculated for whole nuclei are significantly higher in control interphase 14 embryos (99 PIVs from six embryos) than in Colcemid-injected embryos (87 PIVs, five embryos, $P=4.7 \times 10^{-28}$ ), kuk RNAi-depleted embryos (66 PIVs, five embryos, $P=1.7 \times 10^{-7}$ ), or interphase 13 embryos (43 PIVs, four embryos, $P=4.23 \times 10^{-14}$ ). (C') PIV analysis of $\mathrm{H} 2 \mathrm{Av}$ :: GFP in central parts of nuclei reveals higher mean velocities in control IP14 embryos than in Colcemid-treated $\left(P=5.17 \times 10^{-26}\right)$, kuk RNAi $\left(P=5.11 \times 10^{-7}\right)$ or wild-type interphase 13 embryos $\left(P=2.38 \times 10^{-5}\right)$. (D-G) Kymographs of H2AvGFP in one respective nucleus imaged every 2 seconds for 260 seconds in one medial $z$-plane. H2Av::GFP traces appear to be short-lived in nuclei from control interphase 14 embryos (D), whereas they often span the entire movie in interphase 13 nuclei (E), after Colcemid injection (F) or after kuk RNAi (G). Scale bars: $2 \mu \mathrm{m}$ in D-G. (H) Histogram of the residence times of $\mathrm{H} 2 \mathrm{Av}$ ::GFP spots measured from the length of H2Av::GFP tracks as in DG. H2Av::GFP spots from wild-type interphase 14 nuclei (333 spots) remain significantly shorter than in nuclei from wild-type interphase 13 embryos ( 222 spots, $\left.P=8.79 \times 10^{-27}\right)$, Colcemid-injected embryos (300 spots, $\left.P=2.17 \times 10^{-15}\right)$ or $k u k$ RNAi (195 spots, $P=6.89 \times 10^{-12}$ ). 
supplementary material). H2Av::GFP spots were less mobile when NE dynamics was reduced after Colcemid treatment (Fig. 6F) and in interphase 13 (Fig. 6E), or when groove formation was inhibited in $k u k$ mutants (Fig. 6G), as indicated by less ragged traces and longer persistence times (Fig. 6H).

A portion of heterochromatin restricts apically during Drosophila cellularization but not before (Rudolph et al., 2007) (see Fig. S9A,B' in the supplementary material). Chromatin movement due to NE dynamics could therefore contribute to the establishment of chromatin organization. Indeed HP-1-labeled heterochromatin failed to concentrate apically and overlapped with euchromatin upon MT depolymerization (see Fig. S9C-C" in the supplementary material). A similar yet weaker phenotype was seen in kuk embryos (see Fig. S9F-G' in the supplementary material), in agreement with a previous report (Brandt et al., 2006). Nuclei in cellularizing $k u k$ mutants elongated normally but were later to round up (Pilot et al., 2006) (see Fig. S9F,G in the supplementary material). Apical HP-1 restriction failed within the elongation phase (see Fig. S9F, $F^{\prime}$ in the supplementary material), which argues against a mere consequence of reduced nuclear length.

\section{DISCUSSION}

In summary, our results indicate that nuclear shape in Drosophila embryos is not simply determined by nuclear factors that control deformability but instead requires the interplay between active stresses exerted by polymerization of MTs organized in bundles and properties of the NE. Surprisingly, MTs do not shape the NE like a static scaffold that constrains inherent dynamics of the NE. Rather, we find that MT dynamics is essential. Polymerization of MTs produces small high-frequency fluctuations of the NE but is not capable of large-scale deformations into grooves. Groove formation requires MT polymerization within bundles, a property which we show depends on Dynein. We thus propose that pushing forces emanating from MT polymerization events are the fundamental active process underlying nuclear deformations. However, their organization in bundles is essential for lobulation. Bundling of growing MTs along a stationary core probably increases their ability to produce force. In vitro experiments and simulations showed that MTs in a bundle reach pushing forces much higher than the stall force of individual MTs (Laan et al., 2008). Moreover, relaxation of grooves might be facilitated within bundles, as pushing of bundles towards obstacles can facilitate collective catastrophe (Laan et al., 2008). Bundle integrity is ensured by cytoplasmic Dynein. Although we did not detect an enrichment of Dynein at the NE (data not shown), Dynein could be localized at the NE, where it would allow bundle cohesion and attachment. In Drosophila photoreceptors (Patterson et al., 2004) and other systems, Dynein is recruited to the NE by the LINC complex. However, in contrast to Dynein inhibition, reduced activity of the Drosophila LINC complex component CG3287/Klaroid did not affect nuclear morphology. Instead, this enhanced the MT-induced fluctuations of the NE (see Fig. S5B in the supplementary material), indicating that MT polymerization forces are less efficiently buffered. By bridging the NE, LINC proteins are believed to dampen cytoskeletal forces at the lamina or heterochromatin (King et al., 2008). Our observations are consistent with this interpretation.

The LINC complex also transmits cytoskeletal forces across the NE to direct chromosome movement during meiosis (Hiraoka and Dernburg, 2009). In fission yeast, LINC proteins mediate MT- and Dynein-dependant oscillating movements of whole nuclei. This results in nucleoplasmic agitation, which promotes pairing of homologous regions (Chikashige et al., 2007). By analogy, MTinduced fluctuations of the NE could serve as a means to generally enhance chromatin mobility at the onset of zygotic transcription. This would increase the probability of interactions with other loci or the NE and could tune cis-regulatory interactions (Bantignies et al., 2011). The recently established concept of transcription factories where active loci are pulled into pre-assembled sites of mRNA production demands a mobile chromatin (Osborne et al., 2004). Interestingly, transcription is affected in kuk mutants (Brandt et al., 2006), including up- and downregulation of predominantly early zygotic genes. Although a specific role in directly regulating these target genes cannot be excluded, a more likely scenario is a global contribution of Kuk to transcription at this stage owing to its effects on NE mechanics and dynamics as well as chromatin mobility. It will be important to study this quantitatively through direct visualization of dynamics at specific loci and transcription with fluorescence in situ hybridization.

Although necessary, MT polymerization forces are not sufficient to produce grooves in the NE. These deformations require specific material or structural properties of the NE. Our work sheds new light on this process. Comparison of human embryonic stem cells and differentiated cells indicates that deformability is usually increased when stiffness is reduced (and vice versa), for instance owing to absence or knockdown of A-type lamins (Pajerowski et al., 2007; Schape et al., 2009). A Drosophila A-type lamin is not expressed during cellularization, and nuclear deformability is instead controlled by the farnesylated, INM-protein Kuk. Kuk increases the stiffness of the NE, and it is required for large deformations probably because stiffness is required for the pre-stressed NE to buckle. However, Kuk is likely to control other properties of the NE as its depletion cannot be rescued by elevated levels of the B-type lamin Dm0, which also increase NE stiffness. Likewise, overexpressed Dm0 does not enhance lobulation. Kuk could stabilize transient and small deformations imposed by MTs. Stabilization of NE curvature would work as a ratchet and allows the temporal integration of small polymerization forces contributed by individual MTs in bundles. The relative amounts of MT polymerization forces and NE stiffness would define the threshold above which buckling is possible.

\section{Acknowledgements}

We thank our colleagues David Sharp, Roger Karess, Joerg Grosshans, Giacomo Cavalli, Cedric Maurange, Siegfried Roth and Damian Brunner for reagents. We are grateful to J. P. Chauvin for SEM analysis. P. F. Lenne and members of the T.L. group contributed important discussions and comments on the manuscript. This work was supported by the ANR NeMo to T.L. B.H. was a recipient of an EMBO Long-Term Fellowship and is now supported by the ANR NeMo. P.-H.P. and R.F. were supported by the ANR JCJC programme DissecTion.

\section{Competing interests statement}

The authors declare no competing financial interests.

\section{Author contributions}

Experiments were designed by B.H., P.-H.P. and T.L. Y.A.G. performed all FRAP experiments and 3D reconstructions and participated in image analysis. R.F., P.-H.P. and B.H. performed the AFM experiments, O.M. did the PIV analysis and B.H. did all other experiments. The manuscript was written by B.H., P.-H.P. and T.L.

\section{Supplementary material}

Supplementary material for this article is available at

http://dev.biologists.org/lookup/suppl/doi:10.1242/dev.065706/-/DC1

\section{References}

Bantignies, F., Roure, V., Comet, I., Leblanc, B., Schuettengruber, B., Bonnet, J., Tixier, V., Mas, A. and Cavalli, G. (2011). Polycomb-dependent regulatory contacts between distant Hox loci in Drosophila. Cell 144, 214-226. 
Brandt, A., Papagiannouli, F., Wagner, N., Wilsch-Brauninger, M., Braun, M., Furlong, E. E., Loserth, S., Wenzl, C., Pilot, F., Vogt, N. et al. (2006). Developmental control of nuclear size and shape by Kugelkern and Kurzkern. Curr. Biol. 16, 543-552

Brandt, A., Krohne, G. and Grosshans, J. (2008). The farnesylated nuclear proteins KUGELKERN and LAMIN B promote aging-like phenotypes in Drosophila flies. Aging Cell 7, 541-551.

Cavey, M. and Lecuit, T. (2008). Imaging cellular and molecular dynamics in live embryos using fluorescent proteins. Methods Mol. Biol. 420, 219-238.

Chikashige, Y., Haraguchi, T. and Hiraoka, Y. (2007). Another way to move chromosomes. Chromosoma 116, 497-505.

Clarkson, M. and Saint, R. (1999). A His2AvDGFP fusion gene complements a lethal His2AvD mutant allele and provides an in vivo marker for Drosophila chromosome behavior. DNA Cell Biol. 18, 457-462.

Dahl, K. N., Ribeiro, A. J. and Lammerding, J. (2008). Nuclear shape, mechanics, and mechanotransduction. Circ. Res. 102, 1307-1318.

Dauer, W. T. and Worman, H. J. (2009). The nuclear envelope as a signaling node in development and disease. Dev. Cell 17, 626-638.

Dechat, T., Pfleghaar, K., Sengupta, K., Shimi, T., Shumaker, D. K., Solimando, L. and Goldman, R. D. (2008). Nuclear lamins: major factors in the structural organization and function of the nucleus and chromatin. Genes Dev. 22, 832-853

Edgar, B. A., Odell, G. M. and Schubiger, G. (1987). Cytoarchitecture and the patterning of fushi tarazu expression in the Drosophila blastoderm. Genes Dev. 1, 1226-1237.

Franz, C. M., Taubenberger, A., Puech, P. H. and Muller, D. J. (2007). Studying integrin-mediated cell adhesion at the single-molecule level using AFM force spectroscopy. Sci. STKE 2007, pl5

Gonczy, P., Pichler, S., Kirkham, M. and Hyman, A. A. (1999). Cytoplasmic dynein is required for distinct aspects of MTOC positioning, including centrosome separation, in the one cell stage Caenorhabditis elegans embryo. J. Cell Biol. 147, 135-150.

Han, G., Liu, B., Zhang, J., Zuo, W., Morris, N. R. and Xiang, X. (2001). The Aspergillus cytoplasmic dynein heavy chain and NUDF localize to microtubule ends and affect microtubule dynamics. Curr. Biol. 11, 719-724.

Hiraoka, Y. and Dernburg, A. F. (2009). The SUN rises on meiotic chromosome dynamics. Dev. Cell 17, 598-605.

Jankovics, F. and Brunner, D. (2006). Transiently reorganized microtubules are essential for zippering during dorsal closure in Drosophila melanogaster. Dev. Cell 11, 375-385.

King, M. C., Drivas, T. G. and Blobel, G. (2008). A network of nuclear envelope membrane proteins linking centromeres to microtubules. Cell 134, 427-438.

Kracklauer, M.P., Wiora, H. M., Deery, W. J., Chen, X., Bolival, B., Romanowicz, D., Simonetta, R. A., Fuller, M. T., Fischer, J. A. and Beckingham, K. M. (2010). The Drosophila SUN protein Spag4 cooperates with the coiled-coil protein Yuri Gagarin to maintain association of the basal body and spermatid nucleus. J. Cell Sci. 123, 2763-2772.

Laan, L., Husson, J., Munteanu, E. L., Kerssemakers, J. W. and Dogterom, M. (2008). Force-generation and dynamic instability of microtubule bundles. Proc. Natl. Acad. Sci. USA 105, 8920-8925.

Lammerding, J., Schulze, P. C., Takahashi, T., Kozlov, S., Sullivan, T., Kamm, R. D., Stewart, C. L. and Lee, R. T. (2004). Lamin A/C deficiency causes defective nuclear mechanics and mechanotransduction. J. Clin. Invest. 113, 370378

Lammerding, J., Dahl, K. N., Discher, D. E. and Kamm, R. D. (2007). Nuclear mechanics and methods. Methods Cell Biol. 83, 269-294.

Lecuit, T., Samanta, R. and Wieschaus, E. (2002). slam encodes a developmental regulator of polarized membrane growth during cleavage of the Drosophila embryo. Dev. Cell 2, 425-436.
Olins, A. L. and Olins, D. E. (2004). Cytoskeletal influences on nuclear shape in granulocytic HL-60 cells. BMC Cell Biol. 5, 30

Onischenko, E. A., Gubanova, N. V., Kieselbach, T., Kiseleva, E. V. and Hallberg, E. (2004). Annulate lamellae play only a minor role in the storage of excess nucleoporins in Drosophila embryos. Traffic 5, 152-164.

Osborne, C. S., Chakalova, L., Brown, K. E., Carter, D., Horton, A., Debrand, E., Goyenechea, B., Mitchell, J. A., Lopes, S., Reik, W. et al. (2004). Active genes dynamically colocalize to shared sites of ongoing transcription. Nat. Genet. 36, 1065-1071

Pajerowski, J. D., Dahl, K. N., Zhong, F. L., Sammak, P. J. and Discher, D. E. (2007). Physical plasticity of the nucleus in stem cell differentiation. Proc. Natl. Acad. Sci. USA 104, 15619-15624.

Patterson, K., Molofsky, A. B., Robinson, C., Acosta, S., Cater, C. and Fischer, J. A. (2004). The functions of Klarsicht and nuclear lamin in developmentally regulated nuclear migrations of photoreceptor cells in the Drosophila eye. Mol. Biol. Cell 15, 600-610.

Pilot, F., Philippe, J. M., Lemmers, C., Chauvin, J. P. and Lecuit, T. (2006). Developmental control of nuclear morphogenesis and anchoring by charleston identified in a functional genomic screen of Drosophila cellularisation. Development 133, 711-723.

Polychronidou, M., Hellwig, A. and Grosshans, J. (2010). Farnesylated nuclear proteins Kugelkern and lamin Dm0 affect nuclear morphology by directly interacting with the nuclear membrane. Mol. Biol. Cell 21, 3409-3420.

Puech, P. H., Taubenberger, A., Ulrich, F., Krieg, M., Muller, D. J. and Heisenberg, C. P. (2005). Measuring cell adhesion forces of primary gastrulating cells from zebrafish using atomic force microscopy. J. Cell Sci. 118, 4199-4206.

Puech, P. H., Poole, K., Knebel, D. and Muller, D. J. (2006). A new technical approach to quantify cell-cell adhesion forces by AFM. Ultramicroscopy 106, 637-644.

Riemer, D., Stuurman, N., Berrios, M., Hunter, C., Fisher, P. A. and Weber, K. (1995). Expression of Drosophila lamin C is developmentally regulated: analogies with vertebrate A-type lamins. J. Cell Sci. 108, 3189-3198.

Robinson, J. T., Wojcik, E. J., Sanders, M. A., McGrail, M. and Hays, T. S. (1999). Cytoplasmic dynein is required for the nuclear attachment and migration of centrosomes during mitosis in Drosophila. J. Cell Biol. 146, 597-608.

Rudolph, T., Yonezawa, M., Lein, S., Heidrich, K., Kubicek, S., Schafer, C., Phalke, S., Walther, M., Schmidt, A., Jenuwein, T. et al. (2007). Heterochromatin formation in Drosophila is initiated through active removal of H3K4 methylation by the LSD1 homolog SU(VAR)3-3. Mol. Cell 26, 103-115.

Schape, J., Prausse, S., Radmacher, M. and Stick, R. (2009). Influence of lamin $A$ on the mechanical properties of amphibian oocyte nuclei measured by atomic force microscopy. Biophys. J. 96, 4319-4325.

Schejter, E. D. and Wieschaus, E. (1993). Functional elements of the cytoskeleton in the early Drosophila embryo. Annu. Rev. Cell Biol. 9, 67-99.

Sharp, D. J., Rogers, G. C. and Scholey, J. M. (2000). Cytoplasmic dynein is required for poleward chromosome movement during mitosis in Drosophila embryos. Nat. Cell Biol. 2, 922-930.

Stuurman, N., Heins, S. and Aebi, U. (1998). Nuclear lamins: their structure, assembly, and interactions. J. Struct. Biol. 122, 42-66.

Tassy, O., Daian, F., Hudson, C., Bertrand, V. and Lemaire, P. (2006). A quantitative approach to the study of cell shapes and interactions during early chordate embryogenesis. Curr. Biol. 16, 345-358.

Technau, M. and Roth, S. (2008). The Drosophila KASH domain proteins Msp300 and Klarsicht and the SUN domain protein Klaroid have no essential function during oogenesis. Fly 2, 82-91.

Wolf, N., Regan, C. L. and Fuller, M. T. (1988). Temporal and spatial pattern of differences in microtubule behaviour during Drosophila embryogenesis revealed by distribution of a tubulin isoform. Development 102, 311-324 